\title{
Relaxation of energetic S(1D) atoms in Xe gas: Comparison of ab initio calculations with experimental data
}

\section{Citation}

Bovino, S., P. Zhang, V. Kharchenko, and A. Dalgarno. 2011. “Relaxation of Energetic S(1D) Atoms in Xe Gas: Comparison of Ab Initio Calculations with Experimental Data." The Journal of Chemical Physics 135, no. 2: 024304.

\section{Published Version}

doi:10.1063/1.3600352

\section{Permanent link}

http://nrs.harvard.edu/urn-3:HUL.InstRepos:12717323

\section{Terms of Use}

This article was downloaded from Harvard University's DASH repository, and is made available under the terms and conditions applicable to Other Posted Material, as set forth at http:// nrs.harvard.edu/urn-3:HUL.InstRepos:dash.current.terms-of-use\#LAA

\section{Share Your Story}

The Harvard community has made this article openly available.

Please share how this access benefits you. Submit a story.

\section{Accessibility}




\section{AIP Chemicalit Prysics}

\section{Relaxation of energetic S(1D) atoms in Xe gas: Comparison of ab initio calculations with experimental data}

S. Bovino, P. Zhang, V. Kharchenko, and A. Dalgarno

Citation: J. Chem. Phys. 135, 024304 (2011); doi: 10.1063/1.3600352

View online: http://dx.doi.org/10.1063/1.3600352

View Table of Contents: http://jcp.aip.org/resource/1/JCPSA6/v135/i2

Published by the American Institute of Physics.

\section{Related Articles}

Elastic scattering and rotational excitation of nitrogen molecules by sodium atoms J. Chem. Phys. 135, 174301 (2011)

Ultracold $\mathrm{O} 2+\mathrm{O} 2$ collisions in a magnetic field: On the role of the potential energy surface

J. Chem. Phys. 134, 124310 (2011)

Cold and ultracold $\mathrm{NH}-\mathrm{NH}$ collisions: The field-free case

J. Chem. Phys. 134, 124309 (2011)

Quantum and classical study of surface characterization by three-dimensional helium atom scattering J. Chem. Phys. 134, 024319 (2011)

Efficient numerical method for locating Feshbach resonances of ultracold molecules in external fields J. Chem. Phys. 134, 014101 (2011)

\section{Additional information on J. Chem. Phys.}

Journal Homepage: http://jcp.aip.org/

Journal Information: http://jcp.aip.org/about/about_the_journal

Top downloads: http://jcp.aip.org/features/most_downloaded

Information for Authors: http://jcp.aip.org/authors

\section{ADVERTISEMENT}

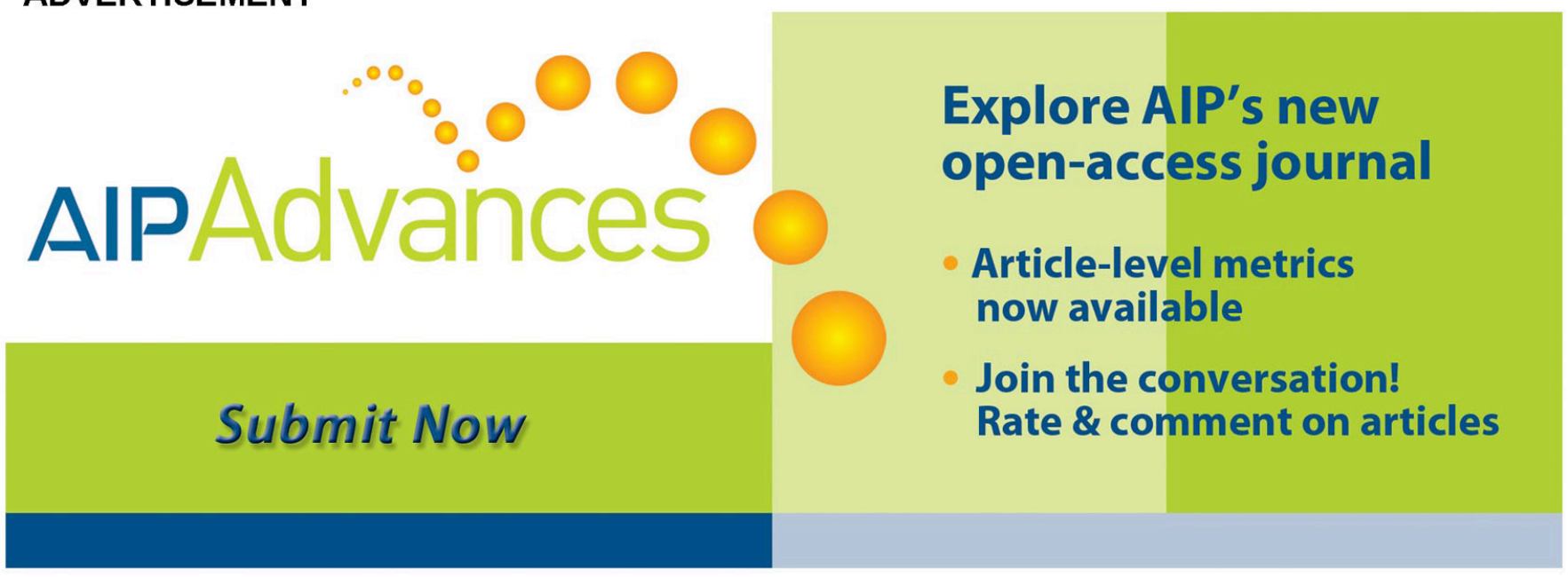




\title{
Relaxation of energetic S( $\left.{ }^{1} \mathrm{D}\right)$ atoms in Xe gas: Comparison of ab initio calculations with experimental data
}

\author{
S. Bovino, ${ }^{1}$ P. Zhang, ${ }^{2}$ V. Kharchenko, ${ }^{2,3}$ and A. Dalgarno ${ }^{2, a)}$ \\ ${ }^{1}$ Department of Chemistry, University of Rome "Sapienza," Rome, Italy \\ ${ }^{2}$ ITAMP, Harvard-Smithsonian Center for Astrophysics, Cambridge, Massachusetts 02138, USA \\ ${ }^{3}$ Department of Physics, University of Connecticut, Storrs, Connecticut 06269, USA
}

(Received 5 April 2011; accepted 25 May 2011; published online 11 July 2011)

\begin{abstract}
In this paper, we report our investigation of the translational energy relaxation of fast $S\left({ }^{1} \mathrm{D}\right)$ atoms in a Xe thermal bath. The interaction potential of Xe-S was constructed using $a b$ initio methods. Total and differential cross sections were then calculated. The latter have been incorporated into the construction of the kernel of the Boltzmann equation describing the energy relaxation process. The solution of the Boltzmann equation was obtained and results were compared with those reported in experiments [G. Nan, and P. L. Houston, J. Chem. Phys. 97, 7865 (1992)]. Good agreement with the measured time-dependent relative velocity of fast $S\left({ }^{1} \mathrm{D}\right)$ atoms was obtained except at long relaxation times. The discrepancy may be due to the error accumulation caused by the use of hard sphere approximation and the Monte Carlo analysis of the experimental data. Our accurate description of the energy relaxation process led to an increase in the number of collisions required to achieve equilibrium by an order of magnitude compared to the number given by the hard-sphere approximation. (C) 2011 American Institute of Physics. [doi:10.1063/1.3600352]
\end{abstract}

\section{INTRODUCTION}

An accurate theoretical description of the translational energy relaxation of energetic atoms and molecules is fundamental to a wide variety of fields of chemical physics, plasma physics, planetary science, and astrophysics. Sources of energetic particles may have very different parameters and character, but the collisional mechanism of energy relaxation is similar in different non-equilibrium systems. The theoretical basis for an accurate description of the energy relaxation process is the Boltzmann kinetic equation. We consider the energy relaxation of fast atoms in an infinite uniform bath gas. If the density of bath gas is much larger than that of the projectile particles, collisions between projectiles may be neglected and evolution of the energy distribution function of fast particles can be described by the linear form of the Boltzmann equation. ${ }^{2}$ Closed analytical expression of the kernel of this Boltzmann equation can be obtained using the fully energy and angular dependent collision cross sections. ${ }^{3}$ The timedependent solution of the Boltzmann equation can then be computed and used for different applications. Successful implementations of this method have been reported in the study of the thermalization problem in physical chemistry, atmospheric physics, and applied atomic spectroscopy. ${ }^{4-6}$

In the present article, we investigate the kinetic energy relaxation of energetic sulfur atoms. Sulfur species have been detected in the upper atmospheres of Jupiter and Titan, ${ }^{7}$ and atomic sulfur is the dominant atmospheric constituent in Jupiter's satellite, Io. ${ }^{8}$ Energetic $S\left({ }^{1} \mathrm{D}\right)$ is produced by the photodissociation of OCS and $\mathrm{H}_{2} \mathrm{~S}$ molecules and the study of energy transfer with other atmospheric gases is important

\footnotetext{
a) Author to whom correspondence should be addressed. Electronic mail: adalgarno@cfa.harvard.edu.
}

for understanding the energy balance of the upper atmosphere and its evolution. Laboratory experiments involving the energy and speed relaxation of $\mathrm{S}\left({ }^{1} \mathrm{D}\right)$ atoms in collisions with planetary and atmospheric gases, such as $\mathrm{N}_{2}, \mathrm{O}, \mathrm{H}$, and $\mathrm{N}$ are challenging, but investigations of $\mathrm{S}$ energy relaxation in rare gases have been carried out. ${ }^{1}$ These experiments concluded that for energy relaxation in heavy bath gases, such as Xe, the effective cross sections, describing the energy transfer collisions, depend strongly on kinetic energy. These conclusions have been drawn from comparisons of the experimental data on the time-dependent energy relaxation of fast $\mathrm{S}$ atoms with the results of Monte Carlo simulations of the $S\left({ }^{1} \mathrm{D}\right)$ thermalization process. The Monte Carlo simulations were carried out using the hard-sphere approximation (HSA) model of atomic collisions. The additional assumption of a completely frozen bath gas was made in these simulations. For collisions of neutral atoms or molecules the HSA is a serious misrepresentation. It does not reproduce the strong forward scattering that arises from the attractive long-range van der Waals interaction and it ignores the energy dependence of the collisional cross sections. For elastic collisions of real atomic particles, the translational energy losses occur by a series of highly probable collisions with relatively small energy transfer occurring in each of these sequential collisions. Therefore, the value of energy transferred per collision and the number of collisions derived by HSA are misleading. We carry out a full theoretical description of the thermalization process by computing accurate angular-dependent cross sections for energy transfer collisions between $\mathrm{S}$ and $\mathrm{Xe}$ atoms and solving the time-dependent Boltzmann equation that describes the energy relaxation. Our calculations, starting with ab initio quantum mechanical calculations of the interaction potential in the $\mathrm{S}$ + Xe system, have no adjustable parameters. The relaxation 
kinetics are characterized by the fast formation and preservation of a quasi-Maxwellian distribution, that occurs for light species thermalized in heavy bath gases (Lorentz gas). ${ }^{5,9}$

In this paper, we report our investigation of the translational energy relaxation of fast $S\left({ }^{1} \mathrm{D}\right)$ atoms in a thermal bath of Xe. The interaction potential of Xe-S is calculated using $a b$ initio methods and the kernel of the Boltzmann equation is constructed employing the fully energy and angular dependent differential cross sections. The solution of the Boltzmann equation is obtained and results are compared with those from experiment. ${ }^{1}$

\section{THEORY}

\section{A. Elastic cross sections}

The energy relaxation of electron volt projectile atoms $\mathrm{S}\left({ }^{1} \mathrm{D}\right)$ in Xe bath gas is dominated by elastic collisions. The Schrödinger equation that governs the dynamics of the colliding particles for center of mass (CM) scattering energy $\varepsilon$ reads

$$
\left[\frac{d^{2}}{d R^{2}}+k^{2}-\left(\frac{2 \mu}{\hbar^{2}}\right) V(R)-\frac{l(l+1)}{R^{2}}\right] u_{l}(R)=0,
$$

where $\mu$ is the reduced mass, $l$ is the angular momentum, $R$ is the internuclear distance, $V(R)$ is the molecular potential, and $k^{2}=2 \mu \varepsilon / \hbar^{2}$. The solution of Eq. (1) at $R \rightarrow \infty$ can be written in terms of the spherical Bessel $\left(j_{l}\right)$ and Neumann $\left(n_{l}\right)$ functions,

$$
u_{l}(R)=k R\left[j_{l}(k R) \cos \eta_{l}-n_{l}(k R) \sin \eta_{l}\right],
$$

where $\eta_{l}$ is the phase shift. At large $R$,

$$
u_{l}(R)=\sin \left(k R-l \pi / 2+\eta_{l}\right),
$$

and the differential cross section is given by

$$
\frac{d \sigma(\varepsilon, \chi)}{d \Omega}=\frac{1}{k^{2}}\left|\sum_{l=0}^{\infty}(2 l+1) \sin \eta_{l} e^{i \eta_{l}} P_{l}(\cos \chi)\right|^{2},
$$

where $P_{l}$ denotes the $l$ th Legendre polynomial and $\chi$ is the $\mathrm{CM}$ scattering angle. The total elastic cross section is

$$
\sigma(\varepsilon)=\frac{4 \pi}{k^{2}} \sum_{l=0}^{\infty}(2 l+1) \sin ^{2} \eta_{l} .
$$

The diffusion or momentum-transfer cross section, which is defined as

$$
\sigma_{d}(\varepsilon)=\frac{4 \pi}{k^{2}} \sum_{l=0}^{\infty}(2 l+1) \sin ^{2}\left(\eta_{l}-\eta_{l+1}\right),
$$

is commonly used for the description of energy transport processes. In the presence of multiple potentials, statistical cross sections are obtained by taking the weighted sum of the individual molecular channels. The statistical weights for the electronic states of the $\mathrm{S}\left({ }^{1} \mathrm{D}\right) \mathrm{Xe}$ molecule, ${ }^{1} \Pi,{ }^{1} \Delta$, and ${ }^{1} \Sigma^{+}$, are $0.4,0.4$, and 0.2 , respectively.

\section{B. Molecular potential: $\boldsymbol{A} \boldsymbol{b}$ initio calculations}

The adiabatic interaction potential between $\operatorname{Xe}\left({ }^{1} S\right)$ and $\mathrm{S}\left({ }^{1} \mathrm{D}\right)$ has three molecular terms, ${ }^{1} \Sigma^{+},{ }^{1} \Pi$, and ${ }^{1} \Delta$. The calculations of these potentials have been performed with the internally contracted multireference configuration interaction with singles and doubles ${ }^{10}$ plus the multireference version of the Davidson correction, ${ }^{11}$ denoted as MRCISD(Q). The reference wave functions of the MRCISD(Q) calculation are obtained from a multiconfiguration self-consistent field (MCSCF) approach. ${ }^{12}$ The active space in the MCSCF includes 14 electrons in 8 molecular orbitals (MOs). The 14 electrons are the valence electrons of $\mathrm{Xe}\left(5 s^{2} 5 p^{6}\right)$ and $\mathrm{S}$ $\left(3 s^{2} 3 p^{4}\right)$ and the $8 \mathrm{MOs}$ are formed from the linear combination of $5 s 5 p$ atomic orbitals of $\mathrm{Xe}$ and $3 s 3 p$ of $\mathrm{S}$. In the $\operatorname{MRCISD}(\mathrm{Q})$ calculations, 32 electrons were correlated. The $4 d$ electrons of $\mathrm{Xe}$ and the $2 s 2 p$ electrons of $\mathrm{S}$ do not participate in the MCSCF active space, but they were correlated through single and double excitations.

A series of Dunning's augmented polarized core/valence aug-cc-pCVnZ basis sets for $\mathrm{S}$ and small-core relativistic pseudo-potential correlation consistent basis sets aug-pVnZPP for Xe ( $n=\mathrm{T}, \mathrm{Q}, 5)$ (Ref. 13) are employed in the MRCISD(Q) calculations. The final potential energies were extrapolated to the complete basis set (CBS) limit by using the mixed exponential/Gaussian function, ${ }^{14}$

$$
V(n)=V_{\mathrm{CBS}}+B e^{-(n-1)}+C e^{-(n-1)^{2}} .
$$

MOLPRO 2006.1 suite of quantum chemistry programs ${ }^{24}$ was used for these electronic structure calculations.

The long-range part of the interaction potential decays as $R^{-6}$. The corresponding dispersion coefficients, $C_{6}$, were evaluated as ${ }^{15}$

$$
C_{6,0}(L)=\frac{3}{\pi} \int_{0}^{\infty} \alpha_{0}(L ; i \omega) \alpha \mathrm{Xe}^{(i \omega) d \omega}
$$

and

$$
C_{6,2}(L)=\frac{3(2 L+3)}{2 \pi L} \int_{0}^{\infty} \alpha_{2}(L ; i \omega) \alpha \mathrm{Xe}^{(i \omega) d \omega,}
$$

where $\alpha_{\mathrm{Xe}}(i \omega)$ is dynamic dipole-dipole polarizability of Xe at imaginary frequency $i \omega$, and $\alpha_{0}(L ; i \omega)$ and $\alpha_{2}(L ; i \omega)$ are the scalar and tensor polarizabilities of $\mathrm{S}$. For ${ }^{1} \mathrm{D},{ }^{16} L=2$ and

$$
\begin{aligned}
& \alpha_{0}(D)=\left[\alpha_{\|}(2,0)+2 \alpha_{\|}(2,1)+2 \alpha_{\|}(2,2)\right] / 5 \\
& \alpha_{2}(D)=\left[-2 \alpha_{\| \mid}(2,0)-2 \alpha_{\|}(2,1)+4 \alpha_{\|}(2,2)\right] / 7 .
\end{aligned}
$$

Here $\alpha_{\|}(L, M)$ refers to $\alpha_{z z}(L, M)$ with the $z$ component as the internuclear axis, $M$ to the projection of the electronic orbit angular momentum $L$ on the $z$ axis. $C_{6}$ for the three singlet electronic states of $\mathrm{XeS}$ are given by

$$
C_{6}(L, M)=C_{6,0}(L)-\frac{3 M^{2}-L(L+1)}{(2 L-1)(2 L+3)} C_{6,2}(L) .
$$

The numerical integration of Eqs. (8) and (9) proceeded with a 50-point Gaussian quadrature. ${ }^{20}$ The dynamic polarizabilities of $S\left({ }^{1} \mathrm{D}\right)$ were obtained from the linear response MCSCF theory ${ }^{17}$ as implemented in DALTON quantum chemistry program. ${ }^{18}$ The active space in this MCSCF calculation is defined as 6 electrons distributed in $13 \mathrm{MOs}$, which include $3 s 3 p 3 d 4 s 4 p$ of S. The d-aug-cc-pCV6Z basis set ${ }^{19}$ was employed in the MCSCF calculation. For Xe, we adopted the 
accurate values obtained from the relativistic many-body calculations in Ref. 20.

\section{Kernel of Boltzmann equation}

The energy evolution of atoms due to elastic and inelastic collisions with a uniform bath gas has been considered by Kharchenko et al. ${ }^{3}$ The rate of energy transfer of the projectile atoms from initial energy $E^{\prime}$ to final energy $E$ in the laboratory frame (LF) is given by the kernel of the Boltzmann equation $B\left(E \mid E^{\prime}\right)$. In a uniform bath gas, whose density is much higher than that of the projectile atoms, collisions and energy exchange between projectile particles are negligible and the energy distribution $f(E, t)$ of the projectile can be described by the linear Boltzmann equation,

$$
\begin{aligned}
\frac{\partial}{\partial t} f(E, t)= & \int B\left(E \mid E^{\prime}\right) f\left(E^{\prime}, t\right) d E^{\prime}-f(E, t) \\
& \times \int B\left(E^{\prime} \mid E\right) d E^{\prime} .
\end{aligned}
$$

For binary elastic and inelastic collisions, $B\left(E \mid E^{\prime}\right)$ can be derived analytically through the double differential cross sections $d^{2} \sigma / d \Omega d \varepsilon^{3}$ and in the case of elastic scattering, the energy relaxation $B\left(E \mid E^{\prime}\right)$ can be calculated using the differential cross section given by Eq. (4),

$$
\begin{aligned}
B\left(E \mid E^{\prime}\right)= & \frac{n_{b} m^{3 / 2}}{2 \sqrt{2} \pi \mu^{2}} \sqrt{E} \\
& \times \int \frac{d \sigma(\varepsilon, \cos \chi)}{d \Omega} \delta\left(E-E^{\prime}+E_{b}\left(\mathbf{p}_{b}^{\prime}+\mathbf{p}-\mathbf{p}^{\prime}\right)\right. \\
& \left.-E_{b}\left(\mathbf{p}_{b}^{\prime}\right)\right) \rho\left(\mathbf{p}_{b}^{\prime}\right) \mathbf{d} \mathbf{p}_{b}^{\prime} \mathbf{d} \Omega_{\mathbf{p}} \mathbf{d} \Omega_{\mathbf{p}^{\prime}},
\end{aligned}
$$

where $m$ is the mass of the projectile, $\Omega_{\mathbf{p}^{\prime}}$ and $\Omega_{\mathbf{p}}$ are the solid angles of the initial $\mathbf{p}^{\prime}$ and final momenta $\mathbf{p}$ in the LF for fixed $\left|\mathbf{p}^{\prime}\right|=\sqrt{2 m E^{\prime}}$ and $|\mathbf{p}|=\sqrt{2 m E}, n_{b}$ is the density of the bath gas, and $\rho\left(\mathbf{p}_{\mathbf{b}}^{\prime}\right)$ is the Maxwell-Boltzmann distribution function of the bath gas. The numerical solution and the propagation scheme used for Eq. (8) have been given in greater detail in earlier publications, ${ }^{4,5}$ and we shall not repeat them. The time-dependent average translational energy is obtained from the time-dependent solution of the Boltzmann equation,

$$
\langle E(t)\rangle=\int f(E, t) E d E,
$$

and the average number of collisions is given by

$$
n(t)=\int_{0}^{t} \int_{0}^{\infty} B\left(E \mid E^{\prime}\right) d E^{\prime} d t^{\prime}
$$

In their paper, Nan and Houston ${ }^{1}$ presented an analysis of their experimental data and obtained the average relative speed of $\mathrm{S}$ and Xe collisions as a function of the number of collisions. The average relative speed $\left\langle v_{i}\right\rangle, \vec{v}_{i}=\vec{v}_{p}-\vec{v}_{b}$, was found by averaging the absolute value of relative velocity over a Boltzmann distribution $f\left(v_{b}\right)$ of the bath gas,

$$
\left\langle v_{i}\right\rangle=\left\langle\left|\vec{v}_{i}\right|\right\rangle=\int_{0}^{\infty} v_{i} f\left(v_{b}\right) d^{3} v_{b}
$$

where $v_{p}$ and $v_{b}$ are velocities of the projectile and the bath gas atoms, respectively. We found

$$
\left\langle v_{i}\right\rangle=v_{T}\left[\sqrt{\frac{2}{\pi}} e^{-\frac{v_{p}^{2}}{2 v_{T}^{2}}}+\operatorname{Erf}\left[\frac{v_{p}}{\sqrt{2} v_{T}}\right]\left(\frac{v_{p}}{v_{T}}+\frac{v_{T}}{v_{p}}\right)\right],
$$

where $v_{T}$ is defined as

$$
v_{T}=\sqrt{\frac{k_{B} T}{m_{b}}}
$$

where $k_{B}$ is the Boltzmann constant, $T$ is the temperature of the bath gas, and Erf is the error function.

\section{RESULTS AND DISCUSSION}

\section{A. Potential energy curves and long-range dispersion coefficients}

We first checked the accuracy of the present $a b$ initio approach by a comparison of the energy separation of $\mathrm{S}\left({ }^{1} \mathrm{D}\right)-\mathrm{S}\left({ }^{3} \mathrm{P}\right)$. The calculated spin-free energy separation is $8989 \mathrm{~cm}^{-1}$. According to Lande's interval rule, the spinorbit coupling (SOC) constant of $\mathrm{S}\left({ }^{3} \mathrm{P}\right)$ is between -177 and $-198 \mathrm{~cm}^{-1}$, obtained from the experimental spin-orbit levels of $\mathrm{S}\left({ }^{3} \mathrm{P}\right){ }^{21}$ Thus, the energy separation of $\mathrm{S}\left({ }^{1} \mathrm{D}\right)-\mathrm{S}\left({ }^{3} \mathrm{P}_{0}\right)$ is 9166-9187 $\mathrm{cm}^{-1}$, which differs from the experiment value of $9238 \mathrm{~cm}^{-121}$ by only $50-70 \mathrm{~cm}^{-1}$. The calculated potential energy curves of the $\mathrm{S}\left({ }^{1} \mathrm{D}\right)-\mathrm{Xe}$ system are shown in Fig. 1. The ${ }^{1} \Sigma^{+}$state is relatively strongly bound and shows a minimum at around $4.60 \mathrm{bohr}$. The ${ }^{1} \Pi$ and ${ }^{1} \Delta$ states are largely repulsive with shallow van der Waals wells in the long range region. Spectroscopic data are listed in Table I for the system with rotational angular momentum $J=0$ corresponding to masses of 131.9041535 amu and 31.97207100 amu for $\mathrm{Xe}$ and S, respectively. A comparison with an earlier theoretical calculation of the excited states of XeS (Ref. 22) is given in Table I. No potential minimum was found for the ${ }^{1} \Delta$ and ${ }^{1} \Pi$ states since the calculations were limited to internuclear distances up to $3.8 \AA$. As for the ${ }^{1} \Sigma^{+}$state, our computed dissociation energy, $D_{0}$, of $0.502 \mathrm{eV}$ is about $0.17 \mathrm{eV}$ deeper than the previous result. A relatively small basis set (double- $\zeta$ quality) was employed in that study.

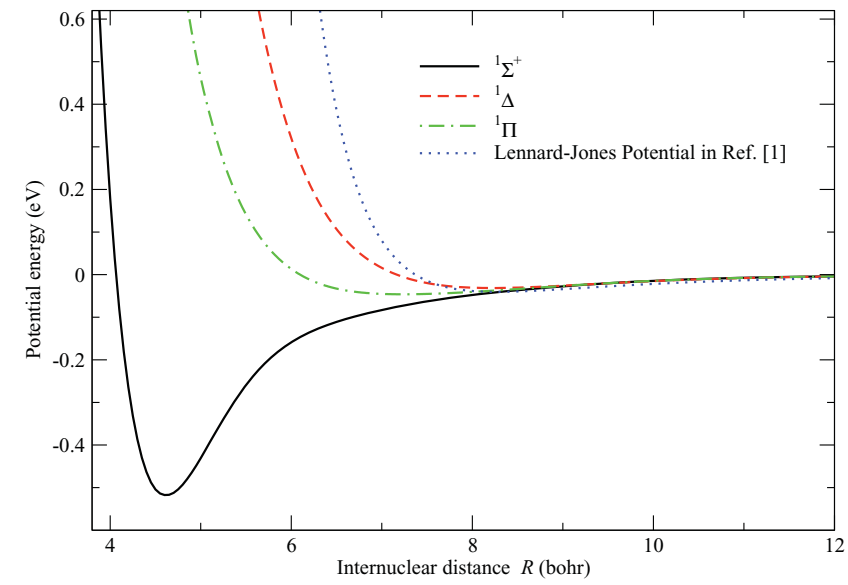

FIG. 1. Diatomic potentials for the three states of S-Xe. 
TABLE I. Spectroscopic constants for the lowest three singlet states of the $\mathrm{XeS}$ molecule.

\begin{tabular}{llccc}
\hline \hline & $D_{0}(\mathrm{eV})$ & $R_{e}(\mathrm{bohr})$ & $\omega_{e}\left(\mathrm{~cm}^{-1}\right)$ & $\omega_{e} x_{e}\left(\mathrm{~cm}^{-1}\right)$ \\
\hline${ }^{1} \Sigma^{+}$(this work) & 0.5020 & 4.60 & 257.9 & 2.18 \\
${ }^{1} \Sigma^{+}$(Ref. 22) & 0.33 & 4.95 & 205 & 2.13 \\
${ }^{1} \Pi$ (this work) & 0.0416 & 7.20 & 32.73 & 0.37 \\
${ }^{1} \Delta$ (this work) & 0.0314 & 8.22 & 32.86 & 0.87 \\
\hline \hline
\end{tabular}

The calculated long-range dispersion coefficients according to Eqs. (8)-(11) are reported in Table II with the derived static dipole polarizabilities of $S\left({ }^{1} \mathrm{D}\right)$. The static dipole polarizabilities have been examined in previous theoretical calculations using the finite field approach with the multireference second order perturbation theory, ${ }^{23}$ and the results are listed in Table II for comparison. Our linear response results are in good agreement with this high-level theoretical analysis; the difference is less than $2 \%$.

\section{B. Elastic differential and total cross sections}

The calculated statistical weighted differential crosssections are shown in Fig. 2 at three selected energies. The oscillating structure of the differential cross sections reflect quantum interference. Large backward scattering is present for low energies. With increasing collision energies, more partial waves contribute to the scattering process and small angle scattering dominates.

The calculated total and diffusion cross sections weighted according to the three potentials are presented in Fig. 3 as a function of the scattering energy. The magnitude of the cross sections is large due to the large polarizabilities of $\mathrm{S}$ and $\mathrm{Xe}$ and strong long-range interaction. The diffusion coefficient $D_{A B}$ can be evaluated from the diffusion cross sections by

$$
D_{A B}=\frac{3 k_{B} T}{16 \mu \Omega_{A, B}} \frac{1}{\left(n_{A}+n_{B}\right)},
$$

where the transport integral $\Omega_{A B}{ }^{26}$ is given in terms of binary collisions between species A and B by

$$
\Omega_{A B}=\frac{2}{\sqrt{\pi \mu}}\left(\frac{1}{2 k_{B} T}\right)^{5 / 2} \int_{0}^{\infty} \varepsilon^{2} \exp \left(-\frac{\varepsilon}{k_{B} T}\right) \sigma_{d}(\varepsilon) d \varepsilon,
$$

where $\sigma_{d}(\varepsilon)$ is given by Eq. (6) and $\left(n_{A}+n_{B}\right)$ is the total molecular number density. $\mathrm{D}_{\mathrm{XeS}}$ can be satisfactorily reproduced for $T \geq 50 \mathrm{~K}$ by using the following expression:

$$
\begin{aligned}
\mathrm{D}_{\mathrm{XeS}}= & -3.701 \times 10^{-12} \times e^{4.9096 / T}+3.323 \times 10^{-15} \\
& +9.264 \times 10^{-13} \times \ln (4.7228 T)
\end{aligned}
$$

TABLE II. Static dipole polarizabilities (a.u.) of $S\left({ }^{1} \mathrm{D}\right)$ and long-range dispersion coefficients (a.u.) of Xe-S $\left({ }^{1} \mathrm{D}\right)$.

\begin{tabular}{lcccc}
\hline \hline State & $\alpha$ (this work) & $\alpha$ (Ref. 23) & State & $\mathrm{C}_{6}$ \\
\hline${ }^{1} \mathrm{D}(\mathrm{M}=0)$ & 18.34 & 18.11 & ${ }^{1} \Sigma^{+}$ & 182.7 \\
${ }^{1} \mathrm{D}(\mathrm{M}=1)$ & 19.37 & 19.18 & ${ }^{1} \Pi$ & 191.0 \\
${ }^{1} \mathrm{D}(\mathrm{M}=2)$ & 22.16 & 22.39 & ${ }^{1} \Delta$ & 216.1 \\
\hline \hline
\end{tabular}

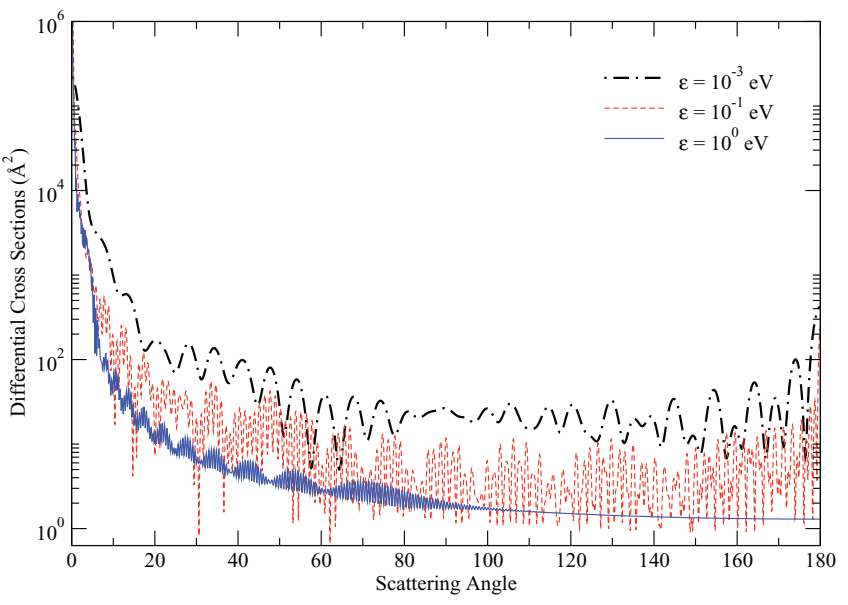

FIG. 2. Differential cross sections at selected CM collision energies $\varepsilon$.

in units of $\mathrm{cm}^{2} \mathrm{~s}^{-1}$.

\section{Energy transfer kinetics}

The calculated differential cross sections were used to construct the kernel of the Boltzmann equation $B\left(E \mid E^{\prime}\right)$ at the experimental bath gas temperatures of $296 \mathrm{~K}$. In Fig. 4, we show the kernels for $T=296 \mathrm{~K}$ and a pressure of 0.947 Torr at three different initial energies $E^{\prime}$ of $0.25,1.20$, and $2.55 \mathrm{eV}$. Sharp maxima with more than four orders of magnitude increase occur at $E \sim E^{\prime}$ because the elastic collisions are dominated by energy losses at small angles. For the time-independent gas density, the kernel scales linearly with the gas pressure.

In the experiment of Nan and Houston, ${ }^{1}$ the fast $S\left({ }^{1} \mathrm{D}\right)$ atoms were produced by $222 \mathrm{~nm}$ photodissociation of the OCS molecule. The Doppler profiles of emission of S atoms were recorded at a fixed delay time of $200 \mathrm{~ns}$ between the photolysis and the probe lasers for different pressures (densities) to determine the time evolution of the relaxation. They adopted HSA and matched these experimental data with Monte Carlo simulations to derive the average relative velocity as a function of the number of collisions. In the Monte

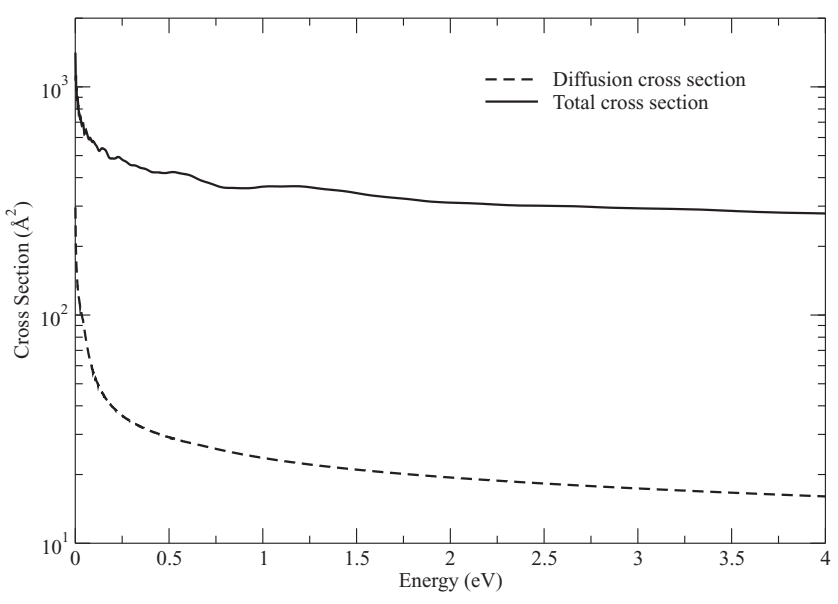

FIG. 3. Total and transport cross sections as a function of CM collision energy. 


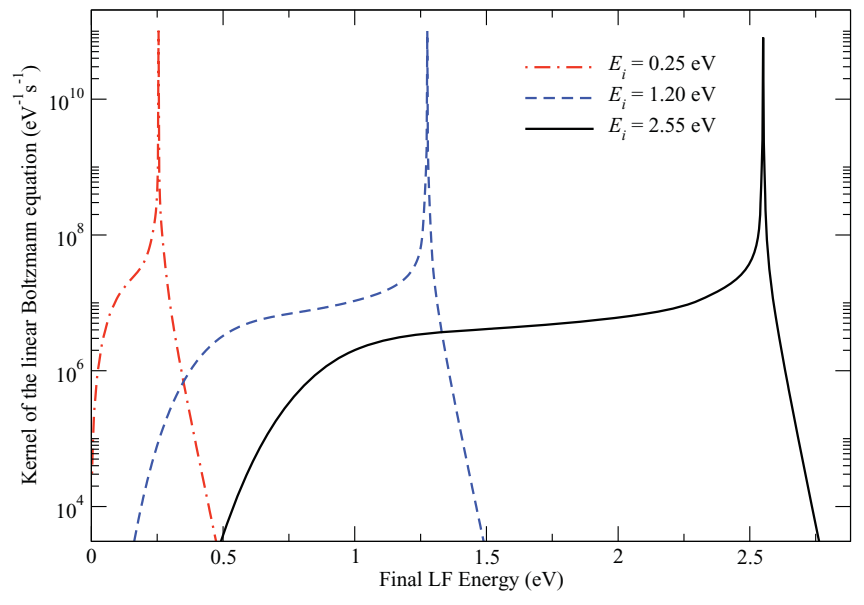

FIG. 4. The kernels of the linear Boltzmann equation at three initial laboratory frame (LF) energies of $E_{i}=0.25, E_{i}=1.20$, and $E_{i}=2.55 \mathrm{eV}$ as a function of the final LF energy. The bath gas temperature is $296 \mathrm{~K}$ and the pressure is 0.947 Torr.

Carlo simulations carried out in Ref. 1, the nascent distribution was constructed by shifting a Maxwellian distribution at $1100 \mathrm{~K}$ by $+600 \mathrm{~m} \mathrm{~s}^{-1}$ and we adopted the same initial distribution of fast atoms. The angular anisotropy is not included in the initial distribution. It is known that the angular relaxation for the Lorentz gas is faster than the energy relaxation, ${ }^{25}$ which is also observed in the experiment of Nan and Houston. The isotropic time-dependent distribution function $f(E, t)$ was obtained by propagating the Boltzmann equation using an efficient matrix exponential scheme. The average relative velocity is obtained from Eq. (17) and the number of collisions from Eq. (15). The calculated results are presented in Table III together with those reported in the experiment. Without any adjustable parameter in our $a b$ initio calculations, the average relative velocities are in close agreement with the results obtained by Nan and Houston. Differences appear at longer relaxation times where our $a b$ initio calculated curve approaches equilibrium faster. This discrepancy may be explained by the strong energy dependence of the relaxation cross sections. In Fig. 5, the average kinetic energy of the evolving sulfur atoms at the experimental bath gas temperature of $296 \mathrm{~K}$ and bath gas pressure of 0.947 Torr, obtained from the solution of the Boltzmann equation, is presented. For comparison, results from HSA with selected

TABLE III. Calculated average projectile velocity $\left(\left\langle v_{\mathrm{S}}\right\rangle, \mathrm{ms}^{-1}\right)$, relative velocity $\left(\left\langle v_{\mathrm{i}}\right\rangle \mathrm{ms}^{-1}\right)$, and number of collisions $\left(n_{\text {col }}\right)$ at selected bath gas pressures (P, Torr). The experimental data are reported in the 4th and 6th columns.

\begin{tabular}{lrrrrc}
\hline \hline $\mathrm{P}$ & $\left\langle v_{\mathrm{S}}\right\rangle$ & $\left\langle v_{i}\right\rangle$ & $\left\langle v_{i}\right\rangle^{\mathrm{a}}$ & $n_{\text {col }}$ & $n_{\text {col }} \mathrm{a}^{\mathrm{a}}$ \\
\hline 0.270 & 1251 & 1266 & 1250 & 47 & $0.6 \pm 0.2$ \\
0.586 & 1062 & 1079 & 1050 & 100 & $1.3 \pm 0.1$ \\
0.947 & 896 & 917 & 910 & 161 & $2.2 \pm 0.2$ \\
1.350 & 761 & 785 & 790 & 226 & $3.5 \pm 0.3$ \\
1.790 & 657 & 686 & 710 & 295 & $4.6 \pm 0.5$ \\
2.780 & 531 & 566 & 647 & 445 & $7.0 \pm 0.9$ \\
\hline
\end{tabular}

${ }^{a}$ Experimental data from Table IV of Ref. 1.

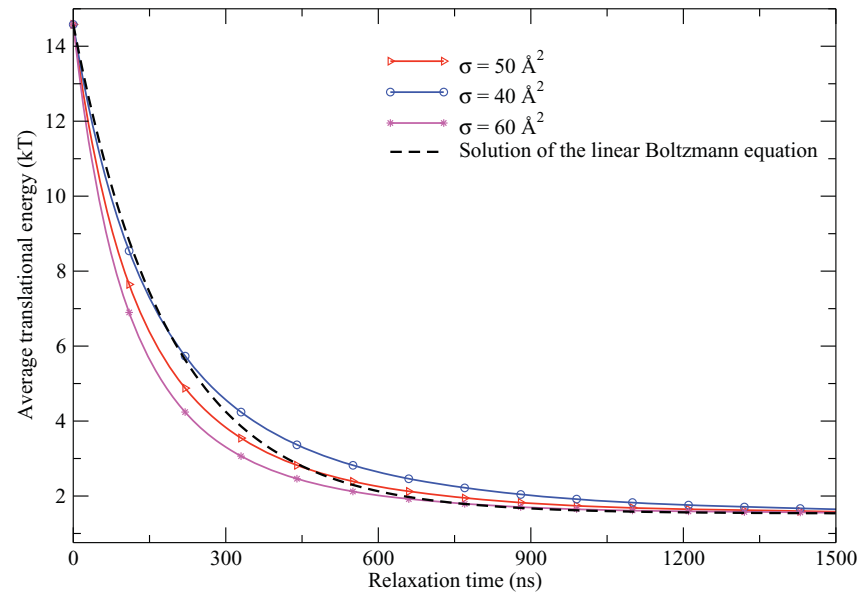

FIG. 5. The translational energy relaxation of sulfur atoms in a Xe bath gas with temperature $T=296 \mathrm{~K}$ and a pressure of 0.947 Torr (dashed curve). The HSA energy relaxation obtained from three different hard sphere cross sections are shown as the curves with symbols.

effective HS cross sections are also shown in Fig. 5. None of the results from HSA reproduce the ab initio calculations. For light particles thermalized in heavy bath gas, as demonstrated earlier, ${ }^{4}$ an energy independent HS cross section is incapable of providing an accurate description of the entire relaxation process. The energy dependence of the effective relaxation cross section was also inferred from the experiment.

It has been shown in previous calculations ${ }^{22}$ that the triplet manifolds, originating from $\mathrm{Xe}-\mathrm{S}\left({ }^{3} \mathrm{P}\right)$, cross with these singlet potential curves in the small $R$ region, and this may lead to the inelastic energy loss because of the SOC. Earlier close-coupling studies ${ }^{27}$ in an analogous system, $\mathrm{Xe}-\mathrm{O}\left({ }^{1} \mathrm{D}\right)$, showed that the cross sections for inelastic SO processes are small, $\sim 20 \AA^{2}$ for collision energies higher than $0.03 \mathrm{eV}$. The $\mathrm{SOC}$ in Xe-S is of the same order of magnitude and may contribute marginally to the translational energy relaxation.

The number of thermalizing collisions obtained from Eq. (15) is at least an order of magnitude larger than those derived by HSA by Nan and Houston. For the elastic scattering of a particle by a long-range interaction potential, the energy transfer per collision, which is determined by the differential cross section or the interaction potentials, is small and a significantly larger number of collisions occurs in comparison with the hard sphere model, in which the cross section is an empirical parameters obtained by fitting the overall rate of energy transfer. For comparison, the Lennard-Jones (LJ) potential extracted from the experimental measurements ${ }^{1}$ is plotted in Fig. 1. The $\mathrm{C}_{6}$ coefficient of the effective potential is 952 a.u. The $\mathrm{LJ}$ potential differs significantly from the actual interactions.

We showed ${ }^{5}$ that the approach to equilibrium in neutral atomic collisions can be described by a two-stage process, the fast formation and preservation of a quasi-Maxwellian distribution and a slow relaxation of the effective temperature of this distribution. We also checked this property in the present research. Figure 6 shows energy distribution functions at selected relaxation times for the same conditions discussed for Fig. 5. The total relaxation time, defined as the time required for the initial energy to relax to the value that is within 

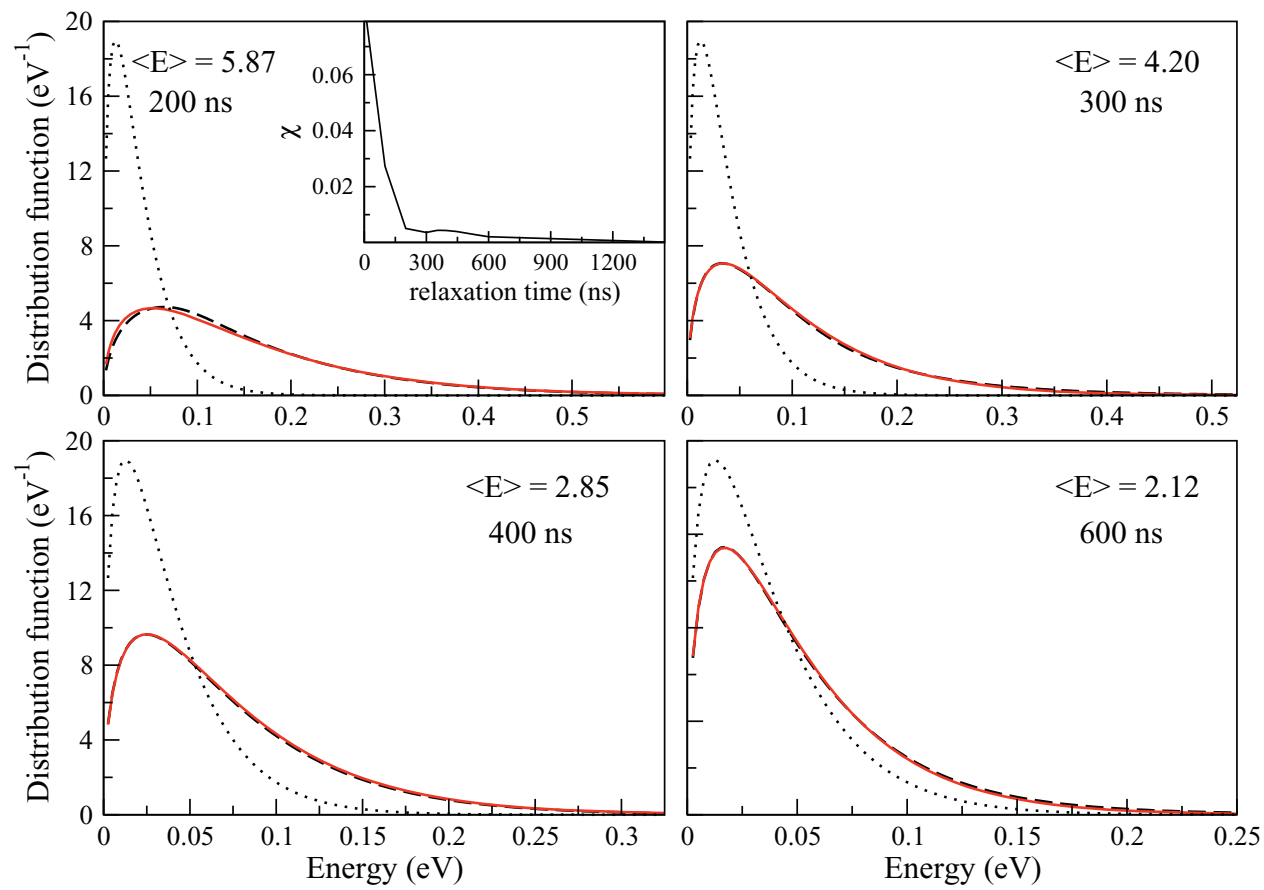

FIG. 6. Calculated energy distribution functions (black dashed curves) at selected relaxation times of fast $\mathrm{S}$ atoms thermalized in Xe gas with temperature of $296 \mathrm{~K}$ and a pressure of 0.947 Torr. The dotted curve represents the Maxwell-Boltzmann distribution of the bath gas. The solid curve is the exact Maxwellian distribution defined according to the effective temperature $T_{\text {eff }}$ at the corresponding relaxation time. The average translational energy, $\langle E\rangle$, is in the units of $k_{B} T$. The embedded picture in the relaxation time at $200 \mathrm{~ns}$ shows the variation of the root mean square deviation, $\chi$, as a function of the relaxation time. $\chi$ reflects the deviation of the time-dependent distribution function from the exact Maxwell-Boltzmann distribution.

$1 \%$ of the thermal energy, is about 1500 ns. The time when the distribution function of the projectile begins to assume a Maxwellian-like shape was numerically determined by its root mean square deviation $(\chi)$ from the exact dimensionless Maxwell-Boltzmann distribution,

$$
f(x)=\frac{2}{\sqrt{\pi}} \sqrt{x} e^{-x},
$$

where $x=E / k_{B} T_{e f f}(t)$ and the effective temperature $T_{e f f}(t)$ is related to the average kinetic energy $\langle E(t)\rangle$ by $T_{e f f}(t)$ $=(2 / 3)\langle E(t)\rangle / k_{B}$. The evolution of $\chi$ is also shown in Fig. 6 . The figure clearly shows that the system reaches a quasi-Maxwellian stage after $300 \mathrm{~ns}$, at which time the average energy is $4.2 k_{B} T$, about three times the thermal energy. The width of the distribution function depends directly on the temperature, and the quasi-Maxwellian distribution is significantly wider than the thermal Boltzmann distributions shown as the dotted curves in Fig. 6.

\section{SUMMARY AND CONCLUSION}

$A b$ initio calculations of the time-dependent thermalization of initially non-thermal sulfur atoms in a buffer gas of xenon were performed. Elastic differential cross sections were evaluated quantum mechanically and used to construct the kernels of the Boltzmann equation, describing the rate of energy transfer collision in the LF. The corresponding time-dependent solutions of the Boltzmann equation were obtained by propagating the LF energy distribution function. Our calculations are compared to the experimental data from Ref. 1. Consistent results for the time dependent relative ve- locity were observed except at long relaxation times. The strong energy dependent relaxation cross sections are responsible for the difference between the results of our $a b$ initio calculations and prediction of the Monte Carlo simulation with the hard sphere model. ${ }^{1}$ The relative velocity reported in the experiment was obtained with the aid of HSA Monte Carlo simulation. $A b$ initio calculations yield a larger number of collisions than HSA to reach equilibrium, reflecting the physical characteristics of the energy transfer process.

\section{ACKNOWLEDGMENTS}

This research of A.D. and P.Z. was supported by the Chemical Science, Geoscience, and Bioscience Division of the Office of Basic Energy Science, Office of Science, (U.S.) Department of Energy (DOE) and of V.K. by NASA Grant Nos. NNX10AB88G and NNX09AF13G. S.B. thanks ITAMP and the Harvard-Smithsonian Center for Astrophysics for his participation in their exchange visitor program.

${ }^{1}$ G. Nan, and P. L. Houston, J. Chem. Phys. 97, 7865 (1992).

${ }^{2}$ I. Oppenheim, K. Shuler, and G. Weiss, Stochastic Processes in Chemical Physics: The Master Equation (MIT, Cambridge, MA, 1977).

${ }^{3}$ V. Kharchenko, J. T. Tharamel, and A. Dalgarno, J. Atmos. Sol.-Terr. Phys. 59, 107 (1997); V. Kharchenko, N. Balakrishnan, and A. Dalgarno, J. Atmos. Sol.-Terr. Phys. 60, 95 (1998).

${ }^{4}$ S. Bovino, P. Zhang, V. Kharchenko, and A. Dalgarno, J. Chem. Phys. 131, 054302 (2009); P. Zhang, V. Kharchenko, M. J. Jamieson, and A. Dalgarno, J. Geophys. Res. 114, A07101 (2009).

${ }^{5}$ P. Zhang, V. Kharchenko, A. Dalgarno, Y. Matsumi, T. Nakayama, and K. Takahashi, Phys. Rev. Lett. 100, 103001 (2008); P. Zhang, V. Kharchenko, and A. Dalgarno, Mol. Phys. 105, 1485 (2007).

${ }^{6} \mathrm{~K}$. Takahashi, N. Taniguchi, Y. Sato, and Y. Matsumi, J. Geophy. Res. 107, 4290 (2002). 
${ }^{7}$ U. von Zhan and D. M. Hunten, Science 272, 849 (1996); H. B. Niemann, S. K. Atreya, G. R. Carignan, T. M. Donahue, J. A. Haberman, D. N. Harpold, R. E. Hartle, D. M. Hunten, W. T. Kasprzak, P. R. Mahaffy, T. C. Owen, N. W. Spencer, and S. H. Way, Science 272, 846 (1996).

${ }^{8}$ M. E. Summers and D. F. Strobel, Icarus 120, 290 (1996).

${ }^{9}$ D. Trunec, P. Španěl, and D. Smith, Chem. Phys. Lett. 372, 728 (2003).

${ }^{10}$ H.-J. Werner and P. J. Knowles, J. Chem. Phys. 89, 5803 (1988).

${ }^{11}$ S. R. Langhoff and E. R. Davidson, Int. J. Quantum Chem. 8, 61 (1974).

${ }^{12}$ H.-J. Werner and P. J. Knowles, J. Chem. Phys. 82, 5053 (1985).

${ }^{13}$ K. A. Peterson, D. Figgen, E. Goll, H. Stoll, and M. Dolg, J. Chem. Phys. 119, 11113 (2003).

${ }^{14}$ K. A. Peterson, D. E. Woon, and T. H. Dunning, Jr., J. Chem. Phys. 100, 7410 (1994); K. A. Peterson and T. H. Dunning, Jr., J. Chem. Phys. 117, 10548 (2002).

${ }^{15}$ A. Dalgarno, Adv. Chem. Phys. 12, 143 (1967); J. P. Hirschfelder and W. J. Meath, Adv. Chem. Phys. 12, 3 (1967).

${ }^{16}$ X. Chu, A. Dalgarno, and G. C. Groenenboom, Phys. Rev. A 72, 032703 (2005).

${ }^{17}$ P. W. Fowler, P. Jørgensen, and J. Olsen, J. Chem. Phys. 93, 7256 (1990).
${ }^{18}$ DALTON, a molecular electronic structure program, Release 2.0, 2005; see http://www.kiemi.uio.no/software/dalton/dalton.html.

${ }^{19}$ J. G. Hill, S. Mazumder, and K. A. Peterson, J. Chem. Phys. 132, 054108 (2010).

${ }^{20}$ A. Derevianko, S. G. Porsev, and J. F. Babb, At. Data Nucl. Data Tables 96, 323 (2010).

${ }^{21}$ Y. Ralchenko, A. E. Kramida, J. Reader, and NIST ASD Team, NIST Atomic Spectra Database, version 4.0.1 (2008).

${ }^{22}$ M. Yamanishi, K. Hirao, and K. Yamashita, J. Chem. Phys. 108, 1514 (1998).

${ }^{23}$ M. Medved, P. W. Fowler, and J. M. Hutson, Mol. Phys. 98, 453 (2000).

${ }^{24}$ MOLPRO, a package of ab initio programs written by H.-J. Werner and P. J. Knowles, R. D. Amos, 2009.1, A. Bernhardsson, A. Berning, et al.

${ }^{25}$ B. Shizgal and R. Blackmore, Chem. Phys. 77, 417 (1983).

${ }^{26} \mathrm{~S}$. Chapman and T. G. Cowling, The Mathematical Theory of NonUniform Gases, 3rd ed. (Cambridge University Press, Cambridge, New York, 1970).

${ }^{27}$ J. S. Cohen, W. R. Wadt, and P. J. Hay, J. Chem. Phys. 71, 2955 (1979). 